\title{
PRESSURE DROP OF BUBBLY TWO-PHASE FLOW IN A SQUARE CHANNEL AT REDUCED GRAVITY
}

\author{
J. F. Zhao, H. Lin, J. C. Xie and W. R. Hu \\ National Microgravity Laboratory, Institute of Mechanics, Chinese Academy of Sciences. \\ 15, Zhong Guan Cun Road, Beijing 100080, China.
}

\begin{abstract}
A new set of experimental pressure drop data, collected aboard the Russian IL-76MDK, is reported for bubbly airwater two-phase flow in a square channel with a cross-sectional area of $12 \times 12 \mathrm{~mm}^{2}$. The present data are compared to several frequently used empirical models, e.g. homogeneous model, Lockhart-Martinelli-Chisholm correlation and Friedel's model. It is shown that the predictions of the models mentioned above are generally not satisfied. A new homogeneous model is developed based on the analysis of the characteristics of bubbly two-phase flow at reduced gravity. It is tested with the present data and other data collected by other researchers in circular pipes. Some questions related to the present model are also discussed. () 2002 COSPAR. Published by Elsevier Science Ltd. All rights reserved.
\end{abstract}

\section{INTRODUCTION}

Two-phase gas/vapor-liquid flows are expected to occur in wide space applications in the future. Reliable design of such systems requires a thorough understanding of the two-phase flow mechanism at reduced gravity condition, such as the phase distributions (flow patterns) in the conduit, the pressure drops and the heat transfer coefficients at various gas and liquid flow rates. These requirements stimulated the studies on two-phase flows in reduced gravity conditions in the past decades, by using the drop tower facilities or parabolic flights of airplanes. There are also simulations of the microgravity two-phase flow in the ground-based experiments, by using the neutral buoyancy method and the two-phase capillary flow system.

The available data of the low gravity pressure drop are scarce, because of the difficulties in obtaining data in parabolic flights, where the gravity rapidly changes and effects the pressure drop transducer sensing lines and pressure drop transducers. In spite of the difficulties, some databases have been established (Zhao and Rezkallah, 1995; Bousman, 1995; Colin and Fabre, 1995).

In the previous experiments on two-phase flow at reduced gravity, circular cross-sectional channels were often used. Non-circular cross-section, however, is often used in heat exchanger, and their circumferences proportional to the cross-sectional area are somewhat better than that of circular tubes. In the latter case, a higher heat transfer rate is possible. A series of experiment on air-water two-phase flow in a square channel with cross-sectional area $12 \times 12 \mathrm{~mm}^{2}$ has been performed aboard the Russian IL-76MDK. Together with the observation of the two-phase flow patterns, the pressure drops were measured in the experiments. In the present paper some of the results, i.e. the two-phase pressure drops of the bubbly flow at reduced gravity are reported and analyzed.

\section{EXPERIMENTAL METHODS}

A schematic diagram of the experimental apparatus is given in Figure 1. The apparatus consists of a test section, a mixer, a liquid flow loop, a gas flow loop, a separator unit, a control and data acquisition system, and a flow pattern-recording camera.

Water and air were used as experimental media. Water was pumped in a closed loop from the separator unit to the test section and back to the separator unit. Water flow rate was varied by adjusting the flow control valve, and was measured using a mass flowmeter with a range of $0-1500 \mathrm{~kg} / \mathrm{h}$ and an accuracy of $0.15 \% \mathrm{FS}$ (full scale). It can also be used to measure the temperature of the fluid. Air was stored in an 8-liter compressed air tank under the 
initial pressure of $6 \mathrm{MPa}$. After the pressure regulator the air pressure was reduced to $0.5 \mathrm{MPa}$. The outlet of the pressure regulator is connected to one of the two circuits for controlling/measuring the gas flow rate. Each circuit consists of a mass flow controller and a solenoid valve. The mass flow controllers have a range of 0-100SLM (standard liters per minute) and 0-10SLM, respectively. The accuracy was $1.5 \% \mathrm{FS}$ for both mass flow controllers. When air mass flow rates were higher than 10SLM, the first circuit was open and the first mass flow controller was used, whilst the second one was closed. Otherwise, the second mass flow controller was used.

The air-water two-phase mixture was supplied through the mixer, where the air is injected radically into water from 50 small holes located uniformly around the periphery of an inner pipe. The outlet of the mixer has the same geometrical shape as the test section with a cross-sectional area of $12 \times 12 \mathrm{~mm}^{2}$. The horizontal test section has a length of $96 \mathrm{~cm}$. The inner wall of the test section consists of transparent Plexiglas. The observation window is located $64 \mathrm{~cm}$ from the mixer outlet and is $15 \mathrm{~cm}$ long. The air-water two-phase flow patterns were recorded by a SONY Hi-8 mm videocamera, operating in a shutter speed of $1 / 4000$ s to reduce the blur in the image.

Absolute pressure was measured at a distant of $18 \mathrm{~cm}$ from the mixer outlet. Two differential pressure measurements were taken at $30 \mathrm{~cm}$ and $81 \mathrm{~cm}$ down stream from the mixer outlet, respectively. All of the pressure readings were taken using Keller pressure transducers with accuracy of $0.25 \% \mathrm{FS}$. The absolute pressure transducer has a range of $0-300 \mathrm{kPa}$, and the ranges for both differential pressure transducers are $0-20 \mathrm{kPa}$. The other sides of the differential pressure transducers were connected to a downstream-facing Pitot tube, which was fulfilled of water and connected to the test tube at $8 \mathrm{~cm}$ up stream from the outlet of the test section. As the system pressure changes, the transducers are equally offset and remain in range. The pressure drop then can be calculated using the difference between the differential pressure transducers. Since the accuracy of the transducers is $0.25 \%$ FS, the uncertainty associated with the measurement of the pressure gradient over the $51 \mathrm{~cm}$ length is $139 \mathrm{~Pa} / \mathrm{m}$.

Two accelerometers were used to measure the g-level during the flights. One accelerometer with a range of $-0.1 \sim 0.1 \mathrm{~g}$ ( $\mathrm{g}$ is the normal gravity on the ground) was used to record the actual $\mathrm{g}$-level in the $\mathrm{x}, \mathrm{y}$ and $\mathrm{z}$ directions during the parabolic flights. The other one has a range of $0-5 \mathrm{~g}$, used to determine the beginning and the ending of the parabolic flights, and to control the experimental processes.

The experiments were performed in reduced gravity conditions on board the IL-76MDK based at the Cosmonaut Training Center of Russia in July 1999. The data of the liquid and gas flow rates, the absolute pressure, the differential pressures, and the liquid temperature were sampled at a rate of $4 \mathrm{~Hz}$ throughout the whole flights. During each parabolic flight period, the data of the differential pressures were also acquired at a sampling rate of $125 \mathrm{~Hz}$. The faster sampled data of the differential pressures were used to calculate the pressure drops. Final data were obtained by averaging of at least 1000 data points.

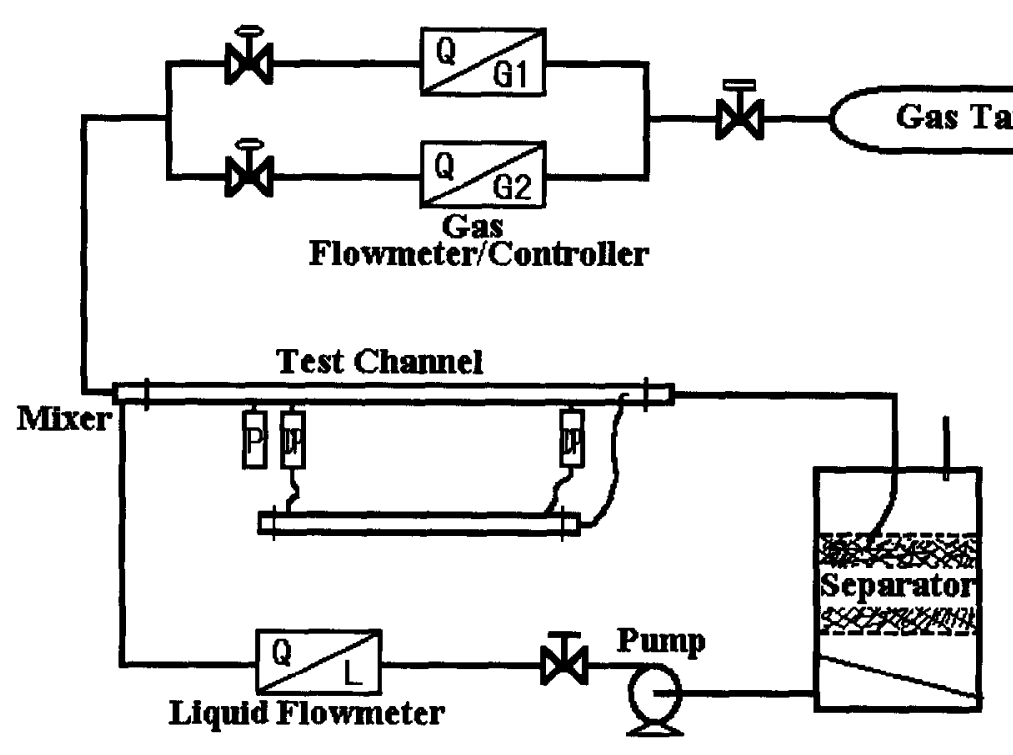

Fig. 1. Scheme of the experimental apparatus. 


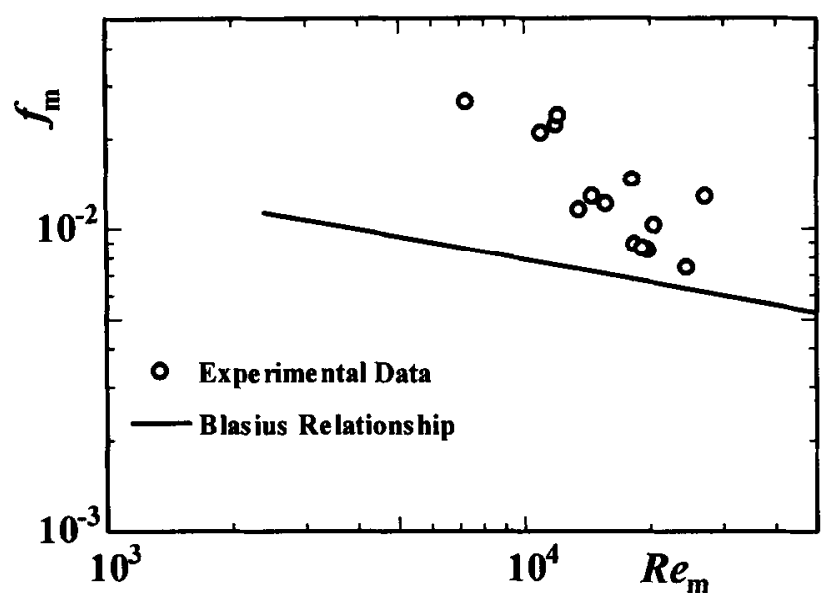

Fig. 2. Comparison between the present experimental data with the homogeneous model.

\section{RESULTS AND COMPARISON WITH EXISTING MODELS}

A number of models are available for predicting the pressure drop of two-phase flows in the terrestrial environments. In the present work the experimental pressure drop data at reduced gravity are compared with some widely used models, e.g. homogeneous model, Lockhart-Martinelli-Chisholm correlation and Friedel's model, to test their validity for microgravity conditions.

The basic assumption of the homogeneous model is that the two phases are well mixed and the velocities of the two phases are equal. This assumption is close to what is actually experienced in the two-phase bubbly flows at microgravity. Therefore, it may be suitable for predicting the pressure drop in those flows.

In the homogeneous model, the relationship between the friction factor $f_{m}=\left[(d p / d z)_{F} D\right] /\left(2 \rho_{m} U_{m}^{2}\right)$ and the mixture Reynolds number $\operatorname{Re}_{m}=\rho_{m} U_{m} D / \mu_{m}$ can be determined from the friction law for single-phase flow, for example, the Blasius relation in the case of turbulent flow through smooth pipes. The mixture density is given by $\rho_{m}^{-1}=x \rho_{G}^{-1}+(1-x) \rho_{L}^{-1}$. The mixture viscosity is given by $\mu_{m}=\mu_{G} \frac{x \rho_{m}}{\rho_{G}}+\mu_{L} \frac{(1-x) \rho_{m}}{\rho_{L}}$, as suggested by Dukler et al. (1964). Figure 2 gives the present experimental data of bubbly two-phase flow. It is shown that the Blasius relation gives much lower predictions for all data.

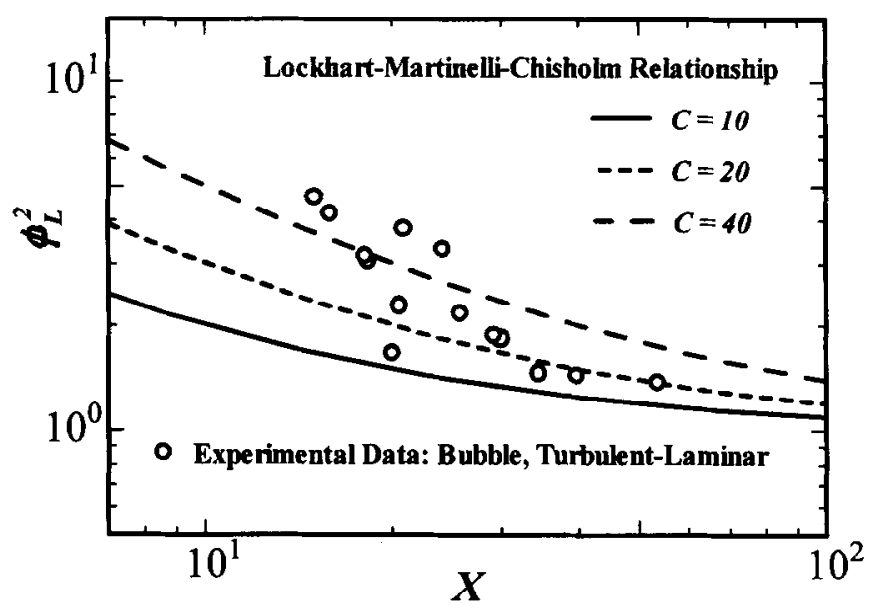

Fig. 3. Comparison between the present experimental data with the Lockhart-Martinelli-Chisholm correlation. 


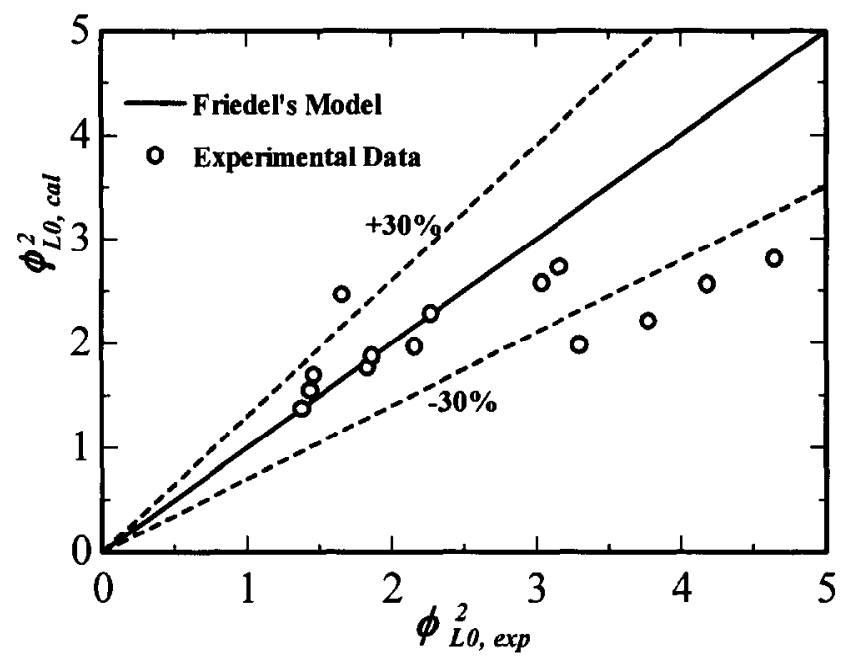

Fig. 4. Comparison between the present experimental data with the Friedel's model.

Another widely used method for estimation of the two-phase pressure drop is the Lockhart-MartinelliChisholm correlation (Chisholm, 1967). The correlation between the liquid two-phase multiplier $\phi_{\mathrm{L}}^{2}$ and the Martinelli parameter $X^{2}$ is given as $\phi_{L}^{2}=1+C / X+1 / X^{2}$, where $C$ is a parameter and its value depends on whether the liquid and gas flows are laminar or turbulent. For example, $C=12$ for the turbulent (liquid) - laminar (gas) case, while $C=21$ for the turbulent (liquid) - turbulent (gas) case. The experimental results and the predictions of the Lockhart-Martinelli-Chisholm correlation with several values of $C$ are plotted against $X$ in Figure 3. It is obviously shown that this correlation is also not good for the present experimental data.

Friedel (1979) proposed an empirical correlation for two-phase pressure drop, in which the effects of the surface tension and the gravity are included. The calculated two-phase multiplier $\phi_{10}{ }^{2}$ cal is compared with the experimental multiplier $\phi_{\mathrm{L} 0}^{2}$ exp in Figure 4 . Generally, the prediction is reasonable, since the deviation of $64 \%$ of the points is less than $30 \%$.

\section{NEW HOMOGENEOUS MODEL}

In the bubbly two-phase flow at microgravity, the gas phase is thought to be mainly concentrated at the tube axis, and then there may exist a gas-free zone near the wall. Therefore, the momentum transfer at the wall may be mainly controlled by the liquid motion. The characteristic values, which are used in the definition of the two-phase friction factor and that of the two-phase Reynolds number, ought to be those of the liquid phase. Based on these argument, Colin et al. (1996) defined the friction factor as $f_{L}=\left[(d p / d z)_{F} D\right] /\left(2 \rho_{L} U_{L}^{2}\right)$, and suggested that the friction factor could be given under the form $f_{L}=F\left(\mathrm{Re}_{L}, \varepsilon\right)$, where $\varepsilon$ is the gas void fraction and $\operatorname{Re}_{L}=\rho_{L} U_{L} D / \mu_{L}$. They didn't give correlation between $f_{L}$ and $\varepsilon$.

However, the liquid averaged velocity $U_{L}$ can not be a good representative of the characteristics of the distribution of the local liquid velocity in the two-phase flows both in terrestrial and in space environments. It is believed that no slip exists between the local velocities of the two phases in bubbly flows at microgravity. The mixture velocity $U_{m}$ will be a better expression for the actual flow of both the liquid and gas phases. Due to the discrepancy between the local velocity distribution and the local void fraction distribution, the mixture velocity is not equal to the liquid averaged velocity. Therefore, the two-phase friction factor and that of the two-phase Reynolds number ought to be defined as $f_{T P}=\left[(d p / d z)_{F} D V /\left(2 \rho_{L} U_{m}^{2}\right)\right.$ and $\operatorname{Re}_{T P}=\rho_{L} U_{m} D / \mu_{L}$.

Using the local velocity distribution determined by Kamp et al. (1993)

$$
u_{m}=u_{0}\left[1-(r / R)^{m}\right] \text {, }
$$




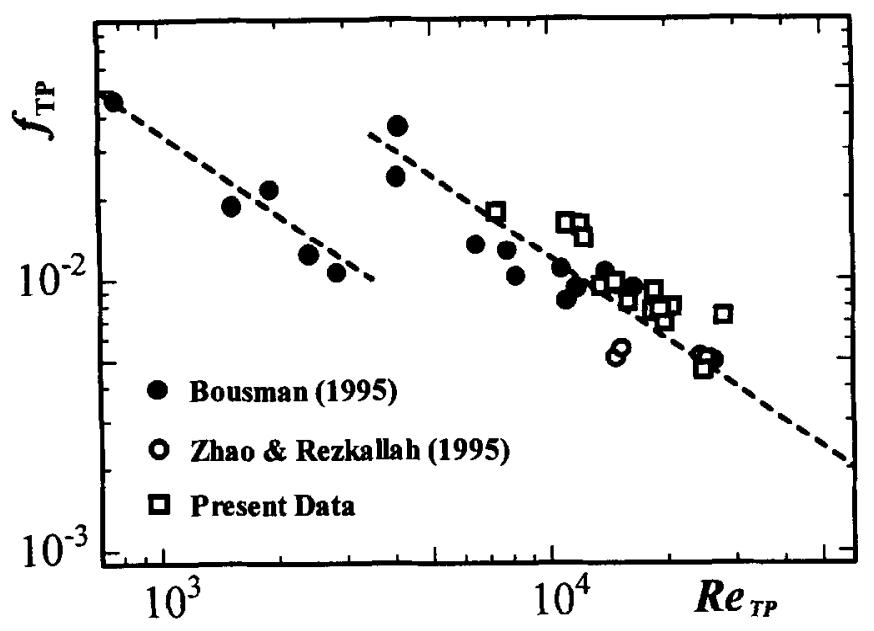

Fig. 5. Comparison between the present model with the experimental data.

and assuming that this form is also coincident with the distribution of the local liquid velocity near the wall, one can obtain the following relationship without any difficulty:

$$
f_{T P}=A \mathbf{R e}_{T P}^{-1},
$$

where $A=4 n(m+2) / m$, and $n$ is the index in the local velocity distribution near the wall.

Generally, the indexes $m$ and $n$ and then the parameter $A$ are all dependent on the Reynolds number and other factors. However, for some ranges of the Reynolds number in which the flow is self-preservative, these indexes may be constants. For example, in fully developed single-phase laminar pipe flows, there is an exactly analytical solution which gives $m=n=2$ and then $A=16$. In the turbulent and/or two-phase flows, there is generally no analytical solution for the velocity distribution. The parameter then must be determined empirically.

The present data of the pressure drop for the bubbly two-phase flow in a square channel at low gravity and those collected by Zhao and Rezkallah (1995) and Bousman (1995) in circular pipes are plotted in Figure5. The dashed lines show results of Eq. (2) with $A=35$ for the case of low Reynolds number and $A=120$ for that of large Reynolds number. It is shown that there may be a transition of flow structure in the range of $3000<\operatorname{Re}_{T P}<4000$, just like the laminar-turbulent transition in the single-phase flow. However, the friction factor of the bubbly twophase flow in the case of low Reynolds number is more than two times as that in the laminar single-phase flow, while in the case of large Reynolds number 7.5 times as that in the laminar single-phase flow. The most obvious difference between the single- and two-phase flows lies upon the Reynolds number's index in the case of large Reynolds number, namely $-1 / 4$ for single-phase flow according to Blasius relationship, while -1 for two-phase flow in the present case. The reason is unknown now.

It is interesting to note that Kamp et al. (1993) found using a least square fit that the value of $m$ equal to 9 for the test corresponding to $U_{S L}=0.77 \mathrm{~m} / \mathrm{s}$ and $U_{S G}=0.044 \mathrm{~m} / \mathrm{s}$ of air-water two-phase flow in a $40 \mathrm{~mm}$-diameter tube $\left(\operatorname{Re}_{T P} \approx 3000\right.$ ). Let $n=m$, one can easily obtain $A=44$. It is only $25 \%$ greater than the present value of $A=35$.

\section{CONCLUSION}

The experiments of two-phase flow through a square channel were conducted onboard the Russian IL76MDK. A new set of experimental pressure drop data for bubbly two-phase flow at reduced gravity is reported. The pressure drop data were compared with the predictions of several widely used empirical models based on the frictional pressure drop data of two-phase flow at normal gravity. In general, the predictions were not good. A new homogeneous model based on the analysis of the characteristics of bubbly two-phase flow at reduced gravity is presented and also compared with the present data, and other data collected by other researchers for circular pipes. Further experimental studies on bubbly two-phase flow at reduced gravity conditions are needed, and a vast amount of pressure drop data needs to be collected still. 


\section{ACKNOWLEDGEMENTS}

This work has been supported by the GESSA (General Establishment for Space Science and Applications) of CAS as a part of the MASE (Microgravity Aircraft Scientific Experiment) program, the Ministry of Science and Technology of China under the grant " $95-Y u-34$ ", the National Natural Science Foundation of China under the grant "19789201", and the Chinese Post-doctoral Science Foundation. The authors also wish to gratefully acknowledge the invaluable assistance of Professor Y. H. Zhang and C. M. Lu of GESSA.

\section{REFERENCES}

Bousman, W.S. Studies of two-phase gas-liquid flow in microgravity. NASA CR 195434, 1995.

Chisholm, D. A theoretical basis for the Lockhart-Martinelli correlation for two-phase flow. Int. J. Heat Mass Transfer. 10, 1767, 1967.

Colin, C. and Fabre, J. Gas-liquid pipe flow under microgravity conditions: influence of tube diameter on flow patterns and pressure drops. Adv. Space Res., 16(7), 137, 1995.

Colin, C., Fabre, J., McQuillen, J., Bubble and slug flow at microgravity conditions: state of knowledge and open questions. Chem. Eng. Comm., 141/142, 155, 1996.

Dukler, A. E., Moye, Wics III and Cleveland, R. G. Frictional pressure drop in two-phase flow. AIChE .J., 10, 38, 1964.

Friedel, L. Improved friction pressure drop correlations for horizontal and vertical two phase pipe flow. In European Two Phase Flow Group Meeting, Paper E2, Ispra, Italy, 1979.

Kamp, A., Colin, C. and Fabre, J. Bubbly flow in a pipe: influence of gravity upon void and velocity distributions. In $3^{\text {rd }}$ World Conference on Experimental Heat Transfer, Fluid Mechanics and Thermodynamics, pp. 14181424, Honolulu, USA, 1993.

Zhao, L. and Rezkallah, K. S., Pressure drop in gas-liquid flow at microgravity conditions. Int. J. Multiphase Flow, 21, 837, 1995. 